\title{
Unfolding of polymers tethered to viscoelastic substrates
}

\author{
Samuel Bell and Eugene M. Terentjev ${ }^{1}$ \\ Cavendish Laboratory, University of Cambridge, J.J. Thomson Avenue, Cambridge, CB3 OHE, \\ U.K.
}

(Dated: 8 August 2019)

The problem of globular polymer unfolding under applied force is a widely-studied fundamental topic in biological and chemical physics, with important applications in cell biology. Much of the existing theoretical and experimental literature focuses on the case where force is applied while fixing the opposite end of polymer chain in space. However, in a realistic biological microenvironment, forces will be applied against viscoelastic references, and the deformation of the folded polymer chain will be combined with the deformation of viscoelastic substrate. In this paper, we consider several simple viscoelastic models for the substrate, and show that its relaxation properties determine the unfolding kinetics. In particular, for low pulling forces, substrates with longer relaxation times cause lower unfolding rates for the pulled polymer chain, whereas for high forces, those substrates with longer relaxation times instead produce higher unfolding rates.

\section{INTRODUCTION}

The problem of a forced breaking of a bond, or unfolding of a globular protein is one of very wide importance across biology, as well as inanimate nature. The dynamic process of life is regulated on the smallest levels by the rates of activated molecular processes underpinning it, whether these be chemical (as in phosphorylation or transcription), electrical (as in ion channels), or mechanical (as in cytoskeletal tension or titin).

The response of biomolecules to mechanical forces has been a popular area of study within biophysics ${ }^{1}$. The field of mechanochemistry is rapidly growing in chemical technology $2 \mid 3$. Increasing sensitivity of experimental tools, like optical tweezers and atomic force microscopy (AFM), and their ability to work in a 'wet' environment, have made them ideal for probing biology with mechanical forces at a molecular level. They have been used extensively to characterise the unfolding kinetics of a range of biomolecules ${ }^{45}$. As well as working with DNA, many experiments have focused on compact globular protein structures, such as the Ig domain, an important subdomain of several proteins, including titin 6 .

AFM experiments are often performed in the positionclamp mode, where the force is measured by the cantilever ${ }^{7}$, or alternatively in the force-clamp mode, where a constant force is applied and the resulting extension measured. These correspond to the Helmholtz and Gibbs ensembles in thermodynamics, respectively. In forceclamp experiments (the Gibbs ensemble), biomolecules typically show abrupt all-or-nothing transitions between folded and unfolded states 11 , meaning that denaturation occurs abruptly and completely once a critical force is reached in the case of force-ramp, or a characteristic time is reached if a constant force is applied.

One interesting topic to which we can apply the ideas of forced unfolding is cell mechanosensation: the transduction of mechanical stimuli from the environment across the cell membrane, and converting them into chemical signals affecting the cell. Mechanosensors are the protein complexes that produce responses to mechanical in- puts $12 \mid 13$. There are two distinct types of mechanosensing: reacting to an external force, or sensing the viscoelastic properties of the cell environment. Here, we refer to the first as mechanosensitivity of the 1st kind, and the latter as mechanosensitivity of the 2 nd kind 14 .

Mechanosensitive ion channels (MSC), such as alamethicin 15 , are an example of mechanosensors of the 1st kind. MSCs exist in all cells and provide a nonspecific response to stress in a bilayer membrane ${ }^{16 \mid 17}$. Traditionally, MSC operation is understood as a twostate model. These two-state systems (open/closed, or bonded/released) with the energy barrier between the states depending on applied force, are common in biophysics 1819. Rates of transition in these systems are often calculated using the 'Bell formula ${ }^{20}$, which has them increasing exponentially with the force. This is just the classical result of Kramers and Smoluchowski21|22, but the application of this formula is not straightforward in the limit of small barriers or high forces.

A mechanosensor of the 2nd kind has a different challenge: to actively measure the response coefficient (elastic stiffness, as in the present work, or matrix viscosity in the case of bacterial flagellar motion). On macroscopic scales (in engineering or rheometry) this is done with two separate measurements: of force (stress) and of position (strain), or we could contrast two separate points of force application. One could also use inertial effects, such as impact or oscillation, to measure the stiffness or elastic constant of the element. None of these options are available on a molecular scale because of a very high resistance (overdamped regime), and of a short-distance cutoff of elasticity. The single molecular sensor complex cannot measure relative displacements in the substrate, and the overdamped dynamics prevents any momentum exchange. Cells must come up with novel ways of measurement. In a recent work 23 , we examined a different paradigm for mechanosensing at integrin adhesion complexes, where the mechanosensing response derives from a conformational change in the protein called the focal adhesion kinase (FAK, see Fig. 1(a) for schematic), which interacts with Rac/Rho signalling pathways, influencing the cytoskeletal dynamics within the cell. There is a clear 


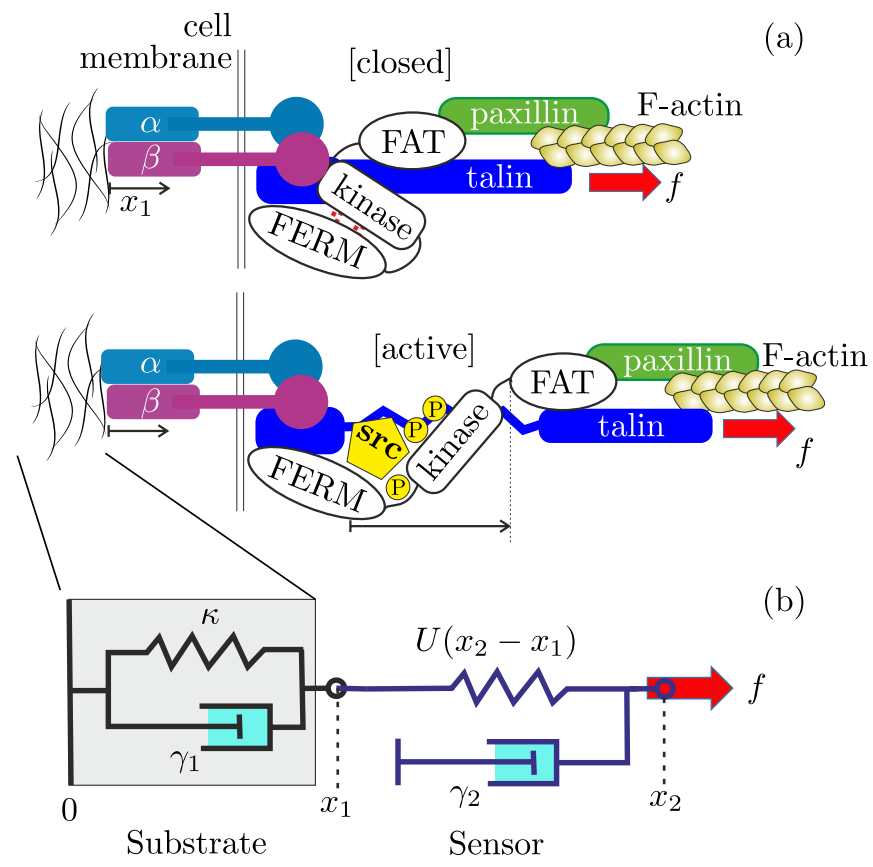

FIG. 1. A schematic of a model focal adhesion complex, and the corresponding mechanical model. (a) The focal adhesion kinase is connected both to a non-rigid extra-cellular matrix (ECM), and to a pulling force through cytoskeletal actin. The FERM domain naturally auto-inhibits the kinase (closed state). On opening, a tyrosine bond is broken between the kinase and FERM domain, allowing the domains to open, and bind Src (the active state), beginning its signalling response. In (b), the viscoelastic substrate is characterised by its elastic stiffness $\kappa$ and the friction coefficient $\gamma_{1}$. The conformational change of FAK is described by a potential $U\left(x_{2}-x_{1}\right)$, see Fig. 2, and the associated relaxation time determined by the damping constant $\gamma_{2}$.

indication that phosphorylation that occurs on unfolding of FAK is the key initial step in the mechanosensing signalling process $\frac{1324}{2}$. We posited that the application of force from the cytoskeleton across FAK is responsible for the conformational change, and used a path approach across the saddle point in the unfolding potential landscape to discuss the unfolding rates as a function of substrate stiffness.

However, the approximation taken in that paper ${ }^{23}$ has some unfortunate limitations. In particular, we found that in the high force regime, the unfolding rates were suppressed, because it takes such a long time for the substrate to equilibrate. This appears attractive from a biological standpoint, as it provides a simple way to ensure a homeostatic response (i.e. a force feedback loop that ensured appropriate cytoskeletal tension). However, from a physical standpoint, this conclusion needs examination. During the entire period of equilibration, our model dictates that some portion of the overall applied force must be present across FAK, or there will be no force applied to the substrate. This force, even if lower, should still increase the unfolding rate as force increases.
In this paper, we consider the same physical system of two coupled stochastic differential equations, see Fig. 1(b), and subject it to new treatment. By first treating certain limiting cases analytically, we develop an idea for the general problem. Then, we change the system variables, and eliminate a redundant degree of freedom, to find an effective diffusion equation for the bond length in the general case. We show that the solution to this can be derived by instead considering the dynamically changing deterministic force across the bond in the absence of stochastic driving forces as a quasi-static force, and applying one-dimensional reaction rate theory.

\section{MODEL}

At its heart, the problem of a bond breaking (globular polymer unfolding) under duress can be captured with two stochastic differential equations. The variables under consideration relate to the position of one end of the polymer anchored in the substrate, $x_{1}$, and the point of force application at the other end of the polymer globule, $x_{2}$. Therefore, the difference in these is effectively the stretching of the bond, up to an additive constant. These variables are subject to separate thermal noise sources, if they are spatially separated: by a flexible linker in the case of single-molecule spectroscopy, and by the cell membrane in the case of cell adhesion. Regarding the bond as harmonic, and the substrate response as viscoelastic, we can write down the pair of Langevin equations describing our system:

$$
\begin{aligned}
& \dot{x}_{1}(t)=-\frac{\kappa}{\gamma_{1}} x_{1}(t)-\frac{1}{\gamma_{1}} \frac{\partial U\left(x_{2}-x_{1}\right)}{\partial x_{1}}+\sqrt{\frac{2 k_{B} T}{\gamma_{1}}} \xi_{1}(t) \\
& \dot{x}_{2}(t)=-\frac{1}{\gamma_{2}} \frac{\partial U\left(x_{2}-x_{1}\right)}{\partial x_{2}}+\frac{f}{\gamma_{2}}+\sqrt{\frac{2 k_{B} T}{\gamma_{2}}} \xi_{2}(t),
\end{aligned}
$$

where $\kappa$ and $\gamma_{1}$ are the elastic and drag response coefficients of the substrate, $\gamma_{2}$ is the measure of dissipation around the bond, $f$ is the force applied to the complex, and $U\left(x_{2}-x_{1}\right)$ is the free energy of the bond, where $u=x_{2}-x_{1}$ is the separation of the bond. The thermal noise terms, $\xi_{1,2}(t)$, are independent normalised Gaussian noise processes.

It is important to be clear how the friction across the bond is implemented in the Langevin equations. The dissipation in the bond is not assumed linked to the cell membrane, which in the language of Fig. 1 is at position $x_{1}$; the bond friction is assumed due to absolute motion of the bond, $\dot{x}_{2}$, rather than relative to the substrate, $\dot{u}=\dot{x}_{2}-\dot{x}_{1}$. For instance, in the case of an AFM pulling experiment, where a protein may be attached to a yielding substrate (a hydrogel or a yielding tether), the friction resisting the absolute motion at $x_{2}$ is a very reasonable assumption, due to the physical separation of the unfolding molecule and the substrate. The case of mechanosensing at focal adhesions in cells is a little 
more nuanced. One might suspect that the motion of cell membrane will create a local reference state for the sensor, and the friction $\gamma_{2}$ responding to this relative motion. However, the cell membrane is highly penetrable to fluids, and its transverse rigidity is negligible. So, motion in the cell membrane will not be directly translated to the sensor environment, and we are free to consider the component of friction coming from the absolute motion of the sensor.

For many biomolecules, there are crucial bonds that have to be overcome before the structure unfolds. If we apply a force, this will bias the free energy in favour of extended configurations. Figure 2(a) shows an example of the expected 'funnel' free energy landscape projected onto the single variable of protein extension. There is a deep minimum, where the fully folded protein sits. Then, once the crucial bonds within the protein break, the protein extends out to its partially unfolded length (often representing the separating domains). Note that there is no unfolding of secondary structure in this phase, and the free energy profile resembles that of an inextensible worm-like chain ${ }^{79 \mid 25}$, quickly diverging at the contour length of the partially unfolded protein. The other way to understand the rapid rise of the free energy at very large extension, even for simple globular homopolymers, is that on complete unfolding all chain segments get exposed to an unfavourable solvent, unable to remain within compact sub-structures. In this paper, we do not consider the large-extension regime, e.g. the worm-like chain behaviour of the protein chain: we are only looking at the breaking of the bond to induce partial unfolding, which in case of FAK would already initiate signalling by phosphorylation of exposed residues. Throughout the text, we choose to model this bond potential as a harmonic potential well of width $u_{m}$, as sketched in Fig. 2(b). We will assume that once the bond reaches the length $u_{m}$, it breaks irreversibly. This obviously need not be true, but it is a simplifying assumption when studying only the breaking rate.

In what follows, we ask about the mean first passage time to the end of the bond influence, $u_{m}$. This is, in general, a very difficult problem that we do not propose to solve fully. First, we consider special cases in which we can solve the system (1) analytically. Then, we use a different reduction of variables, by considering the mean weight at a fixed distance from the line of first passage, and then using the distance from the line of first passage as our variable.

\section{Estimates of material parameters}

We shall find that our model predictions are very sensitive to values of several key parameters, so a careful discussion of their estimates is required.

We start with the strength of the bond holding the FERM and kinase domain in the closed (inhibited) state, labeled as $\Delta G_{\mathrm{o}}$ in Fig. 22 The MD simulation study 26

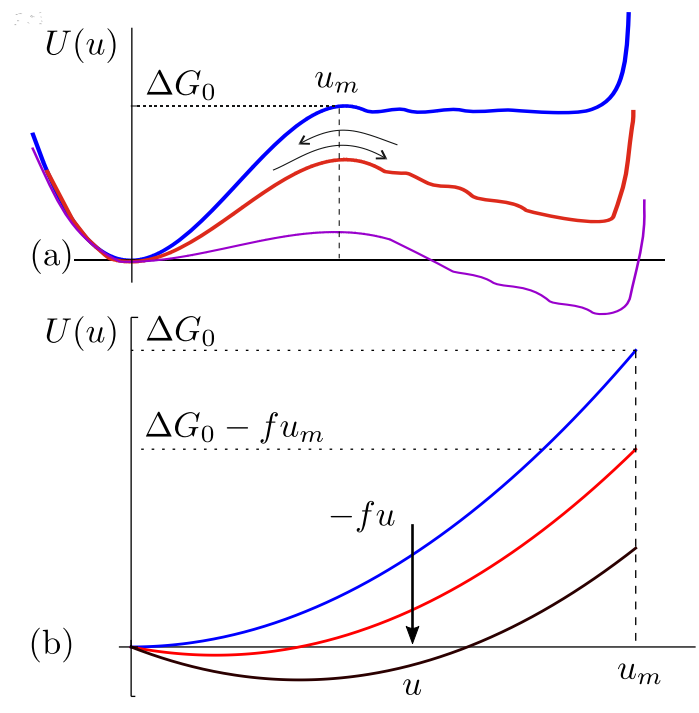

FIG. 2. Modelling a bond potential under force: a schematic of (a) the full FAK potential with a long plateau as the FERM and kinase domains separate until the tether between them is at full stretch, where the potential resembles a worm-like chair 25 , and (b) the model harmonic potential $U=\mu u^{2} / 2$. For the model potential in (b), once the bond length $u=x_{2}-$ $x_{1}$ reaches a critical value, $u_{\mathrm{m}}$, it will snap open irreversibly. Increasing the force applied to the bond lowers the barrier height and shifts the minimum closer to the barrier position.

estimated the energy barrier for FAK opening as $\Delta G_{\mathrm{o}} \approx$ $28.5 k_{B} T$, which is $17 \mathrm{kcal} / \mathrm{mol}$ at room temperature. This value seems too high, and the authors o[ ${ }^{26}$ also comment on that. It is known that interdomain hydrophobic interaction in such proteins is usually low-affinity. For instance, a measurement in a different multi-domain protein gives a value for this bonding energy is $7 \mathrm{kcal} / \mathrm{mol}$, or $\sim 11 k_{B} T^{27}$. However, this is close to an energy of just 1-2 hydrophobic contacts, and there is more affinity between FERM and kinase domains observed in 26 . In the end, we select an intermediate value between the two limits mentioned above: $\Delta G_{\mathrm{o}} \approx 17 k_{B} T$, or $10 \mathrm{kcal} / \mathrm{mol}$.

The position of the barrier in FAK can be obtained directly from the computational study: $u_{m}=0.9 \mathrm{~nm}^{26}$, which is a reasonable value for the protein domain structure. This allows to determine the value of critical force at which the native minimum disappears altogether, and the closed (foded) state becomes completely unstable, $f_{c}=2 \Delta G_{\mathrm{o}} / u_{m} \approx 150 \mathrm{pN}$. This is a very high force that is likely to unfold most proteins, and is also unlikely to be generated by a single actin filament of a cell cytoskeleton. For comparison, studies investigating the force required to disrupt the fibronectin-integrin-cytoskeleton linkage, report the value of only 1-2 $\mathrm{pN}^{28 / 29}$; this, in turn, is probably too low (an underestimate) since a single myosin motor exerts $\sim 3 \mathrm{pN}$ of force ${ }^{30131}$. So we should explore the effect of pulling forces in the range of single to tens of $\mathrm{pN}$.

Let us now look at the substrate stiffness. For reference, the elastic modulus of a collagen-rich mammalian 
tendon is: $1.2 \mathrm{GPa}$, of a collagen/elastin ligament: $1.1 \mathrm{Mpa}^{33}$, and of an aorta wall: $0.8 \mathrm{MPa}{ }^{34}$. Synthetic rubber has a modulus around $100 \mathrm{kPa}$. Epithelial and glial tissues have a much lower modulus: $100 \mathrm{~Pa}-$ $1 \mathrm{kPa} 36 \mid 37$. If a half-space occupied by an elastic medium (e.g. gel substrate or glass plate) with the Young modulus $Y$, and a point force $f$ is applied along the surface (modeling the pulling of the integrin-ECM junction, Fig. 1a)), the response coefficient (spring constant) that we have called the 'stiffness' is given by $\kappa=(4 / 3) \pi Y \xi$, where $\xi$ is a short-distance elastic cutoff: a length scale analogous to the mesh size of a densely packed (nonfilamentous) substrate. This is a classical relation going as far back as Lord Kelvin 38. In the work of Janmey et

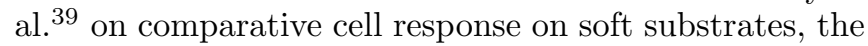
weakest substrate had $Y=540 \mathrm{~Pa}$. For a more typical weak gel with $Y=10 \mathrm{kPa}$, and a characteristic network mesh size $\xi=10 \mathrm{~nm}$, we obtain $\kappa=4.2 \cdot 10^{-4} \mathrm{~N} / \mathrm{m}$. On a stiff mineral glass with $Y=10 \mathrm{GPa}$, we must take the characteristic size to be a 'cage' size (slightly above the size of a monomer), $\xi=1 \mathrm{~nm}$, which gives $\kappa=42 \mathrm{~N} / \mathrm{m}$. A typical stiff plastic has a value about 10 times smaller. So a large spectrum of stiffnesses $\kappa$ could be explored by living cells.

Finally, we need estimates of the damping constants. The simulation study 26 determined a very reasonable value for the internal diffusion constant of the FAK complex: $D=k_{B} T / \gamma_{2} \approx 6 \cdot 10^{-12} \mathrm{~m}^{2} \mathrm{~s}^{-1}$. At room temperature, this gives the damping constant: $\gamma_{2}=$ $7 \cdot 10^{-10} \mathrm{~kg} \mathrm{~s}^{-1}$. Then, the overall scale ('bare magnitude') of the FAK opening rate derived below, Eq. 22, is approximately $\left(2 \Delta G_{\mathrm{o}} / u_{m}^{2} \gamma_{2}\right) \approx 1.6 \cdot 10^{8} \mathrm{~s}^{-1}$, which means a time scale of around 6 ns. This 'bare' time scale is compatible with available data and simulations on full and partial protein unfolding 40 ; naturally, at given bonding energy and low pulling force the actual rate of FAK opening/activation would be much lower: the plots below suggest tens of microseconds to milliseconds range.

To estimate the damping constant of the viscoelastic substrate, we need the characteristic time of its internal relaxation, which we define as $\tau_{1}=\gamma_{1} / \kappa$ in our notation. It is important to note that the local time of relaxation of thermal fluctuations must not be confused with the macroscopic stress relaxation time, which can sometimes be very long in rubbers and gels. To estimate this, we use a study conducted using both AFM and a classical rheometer on a variety of polyacrylamide gels 41 . They found a reasonably constant value of $\tau_{1}=2 \cdot 10^{-4} \mathrm{~s}$ across gels from $300 \mathrm{~Pa}$ to $10 \mathrm{kPa}$. Therefore, we take $\gamma_{1}=$ $\tau_{1} \kappa$. As such, the ratio between the damping coefficients, $\gamma_{2} / \gamma_{1}$, takes a wide range of values, from 0.2 for soft substrates, to $10^{-4}$ for stiffer substrates.

\section{ORNSTEIN-UHLENBECK APPROACH}

Instead of considering the whole free energy profile of our extending biomolecule, Fig. 2(a), we can ask about the mean first passage time to the end of the harmonic section of potential, so that we may consider the potential $U\left(x_{2}-x_{1}\right)=\mu\left(x_{2}-x_{1}\right)^{2} / 2$ as in Fig. 2(b). One can re-write out the system in Eqs. (1) in a standard vector form, for $\boldsymbol{x}(t)=\left(x_{1}(t), x_{2}(t)\right)$, and stochastic force $\boldsymbol{\xi}(t)=$ $\left(\xi_{1}(t), \xi_{2}(t)\right.$ :

$$
\dot{\boldsymbol{x}}=-M(\boldsymbol{x}(t)-\overline{\boldsymbol{x}})+\sigma \boldsymbol{\xi}(t),
$$

where the constant parameters $M, \overline{\boldsymbol{x}}, \sigma$ are defined as:

$$
\begin{aligned}
M & =\left(\begin{array}{cc}
(\kappa+\mu) / \gamma_{1} & -\mu / \gamma_{1} \\
-\mu / \gamma_{2} & \mu / \gamma_{2}
\end{array}\right), \quad \overline{\boldsymbol{x}}=M^{-1}\left(\begin{array}{c}
0 \\
f / \gamma_{2}
\end{array}\right), \\
\sigma & =\left(\begin{array}{cc}
\sqrt{2 k_{B} T / \gamma_{1}} & 0 \\
0 & \sqrt{2 k_{B} T / \gamma_{2}}
\end{array}\right) .
\end{aligned}
$$

Physically, the matrix $M$ gives the coefficients of restoring force, while the matrix $\sigma$ characterises the strength of thermal fluctuations in the system. The vector $\overline{\boldsymbol{x}}$ gives the equilibrium position of the system in the absence of thermal fluctuations. Equation (2) is the standard form of a two-dimensional Ornstein-Uhlenbeck process. The stochastic force matrix $\sigma$ need not be diagonal, but in the set-up of our problem in $\left(x_{1}, x_{2}\right)$ variables, it is. One can solve this problem by finding the normal modes of the system, which will be one-dimensional OrnsteinUhlenbeck processes, $\boldsymbol{x}^{\prime}=R^{-1} \boldsymbol{x}$, where $R$ is the matrix of eigenvectors. Now, the thermal noise matrix, $R^{-1} \sigma$ is not necessarily diagonal, so the two normal modes, while dynamically independent processes, are still going to be coupled through their stochastic driving forces. However, if one of these normal modes is proportional to the bond separation $u: x_{i}^{\prime} \propto x_{2}-x_{1}$, then we can ignore the other normal mode, and treat the bond separation as a single one-dimensional Ornstein-Uhlenbeck process.

There are two cases where the problem naturally reduces easily to one variable: the case of $\kappa \rightarrow \infty$, when the substrate is completely rigid, and $x_{1}$ is fixed, and the case that $\kappa=0$, when the anchoring substrate has no elastic modulus, and only the damping remains. In these cases, one can quickly arrive at expressions for the mean time of passage to $u_{m}$, using a known result derived in the important paper by Ricciardi and Satd $\underline{42}$. That paper takes a one-dimensional Ornstein-Uhlenbeck process written in a standard form, $\dot{x}(t)=-M x(t)+\sigma \xi(t)$, starting at $x(0)$, and reaching a final value $S>x(0)$. Then, the mean first-passage time (MFPT) is given by

$$
\tau=\frac{1}{2 M}[\phi(S)-\phi(x(0))],
$$

where the dimensionless function $\phi(z)$ is the infinite series

$$
\phi(z)=\sum_{n=1}^{\infty} \frac{\Gamma(n / 2)}{n !}\left(\frac{z \sqrt{M}}{\sigma}\right)^{n}
$$

In the case that $0<S<x(0)$, one should take the problem with $S \rightarrow-S$ and $x(0) \rightarrow-x(0)$. This careful treatment of signs is necessary as the initial conditions 


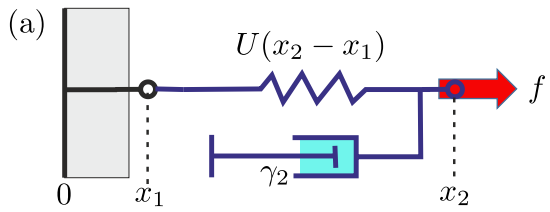

Rigid substrate Sensor

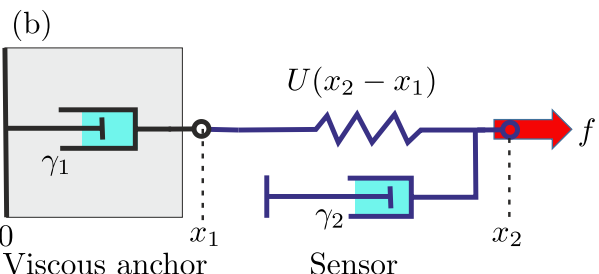

FIG. 3. Schematics showing the two limiting cases in which the problem reduces to a single variable of the bond separation $u$. (a) The rigid substrate case, where $\kappa \rightarrow \infty$, and (b) the viscous anchor chase, where $\kappa \rightarrow 0$.

explored are force-dependent, and so depending on the value of the force we may have to use different expressions. Finally, in the case where the target boundary $S$ is sufficiently far away from the origin that the memory of the initial condition $x(0)$ can be considered lost, Ricciardi and Satc 22 give a compact asymptotic expression for the MFPT:

$$
\tau \approx \sqrt{\frac{\pi \sigma^{2}}{M^{3}}} \frac{1}{S} \exp \left[\frac{M S^{2}}{\sigma^{2}}\right] .
$$

\section{A. Rigid substrate}

In this case, when $\kappa \rightarrow \infty$, the value of $x_{1}$ is constant and the Eqs. (1) reduce to the single dynamical equation for $x_{2}$, and so the equation determining the separation $u=x_{2}-x_{1}$ takes the form:

$$
\dot{u}=-\frac{\mu}{\gamma_{2}}\left(u-\frac{f}{\mu}\right)+\sqrt{\frac{2 k_{B} T}{\gamma_{2}}} \xi(t)
$$

We are seeking the MFPT from $u_{0}=0$ to the final bond length $S=u_{m}>0$. To put Eq. (7) into the RicciardiSato form, we shift the variable: $\widetilde{u}=u-f / \mu$. The function $\phi(z)$ has the form:

$$
\phi(z)=\sum_{n=1}^{\infty} \frac{\Gamma(n / 2)}{n !}\left(z \sqrt{\frac{\mu}{2 k_{B} T}}\right)^{n}
$$

Then, as in Eq. (4), the MFPT is the difference between the values of this function between two limits of $z$ : starting at $\tilde{u}(0)=-f / \mu$, and finishing at $\tilde{S}=u_{m}-f / \mu$.

$$
\tau_{\text {rigid }}=\frac{\gamma_{2}}{2 \mu}\left[\phi\left(u_{m}-f / \mu\right)-\phi(-f / \mu)\right] .
$$

This result holds while there is still a stable minimum at $\tilde{u}(0)$ in the potential. This will be true for forces less than the critical force $f_{c}=\mu u_{m}$, where the minimum will coincide with the barrier position $u_{m}$.

\section{B. Viscous anchor}

When the substrate has no elastic modulus, and is instead only a viscous anchor, we can set $\kappa=0$ in Eq. (2). The inverse of the matrix of eigenvectors reduces to a simple form:

$$
R^{-1}=\frac{\gamma_{1}}{\gamma_{1}+\gamma_{2}}\left(\begin{array}{cc}
1 & \gamma_{2} / \gamma_{1} \\
-1 & 1
\end{array}\right)
$$

The second normal mode, $x_{2}^{\prime}=\left(x_{2}-x_{1}\right) \gamma_{1} /\left(\gamma_{1}+\gamma_{2}\right)$, is just the bond separation $u$ scaled by a constant factor. However, unlike the case of a completely rigid substrate, here we have to deal with thermal fluctuations both in the substrate and in the bonded complex. For a viscous anchor, the full solution for our natural variable $u$ is:

$$
\begin{aligned}
u(t) & =u_{0} e^{-\lambda t}+\frac{f}{\mu}\left(1-e^{-\lambda t}\right) \\
& -\int_{0}^{t} e^{-\lambda(t-s)}\left[\sqrt{\frac{2 k_{B} T}{\gamma_{1}}} \xi_{1}(s)-\sqrt{\frac{2 k_{B} T}{\gamma_{2}}} \xi_{2}(s)\right] d s .
\end{aligned}
$$

The eigenvalue $\lambda=\mu\left(\gamma_{1}+\gamma_{2}\right) / \gamma_{1} \gamma_{2}$ is the relaxation rate of the normal mode, since there is a bond stiffness $\mu$ divided by an effective friction coefficient $\tilde{\gamma}=\gamma_{1} \gamma_{2} /\left(\gamma_{1}+\right.$ $\left.\gamma_{2}\right)$. We can combine the two stochastic integrals, using a version of the Pythagoras theorem, by writing the two white noise terms in Eq. (11) as:

$$
\begin{aligned}
\sqrt{\frac{2 k_{B} T}{\gamma_{1}}} \xi_{1}(t)+\sqrt{\frac{2 k_{B} T}{\gamma_{2}}} \xi_{2}(t) & =\sqrt{\frac{2 k_{B} T}{\gamma_{1}}+\frac{2 k_{B} T}{\gamma_{2}}} \xi(t), \\
& =\sqrt{\frac{2 k_{B} T}{\tilde{\gamma}}} \xi(t) .
\end{aligned}
$$

It is clear that upon substitution into Eq. (11), we get a one-dimensional Ornstin-Uhlenbeck process. To use the Ricciardi-Sato formula, we must again shift into coordinates $\tilde{u}=u-f / \mu$. The only difference between the rigid substrate limit and the case of a viscous anchor is the substitution of the bond dissipation constant $\gamma_{2}$ for the reduced dissipation constant $\tilde{\gamma}$ in front, so that the MFPT for forces less than the critical force, $f<\mu u_{m}$, is again of the form:

$$
\tau_{\text {visc }}=\frac{\tilde{\gamma}}{2 \mu}\left[\phi\left(u_{m}-f / \mu\right)-\phi(-f / \mu)\right],
$$

where the function $\phi(z)$ is given in Eq. (8). Immediately we see that if there is very high dissipation in the viscous anchor, then $\tilde{\gamma} \rightarrow \gamma_{2}$, and the system behaves as if the substrate is rigid.

Figure 4(a) shows that the MFPT (plotted on a logarithmic scale) decreases as a function of applied force. For a fixed barrier height $\Delta G_{\mathrm{o}}$, stiffer bonds would be faster to break, as the barrier is closer to the potential minimum. The mean first-passage time for a viscous anchor is plotted in Fig. 4(b). The larger the friction coefficient of bond fluctuations, the longer the unbinding 

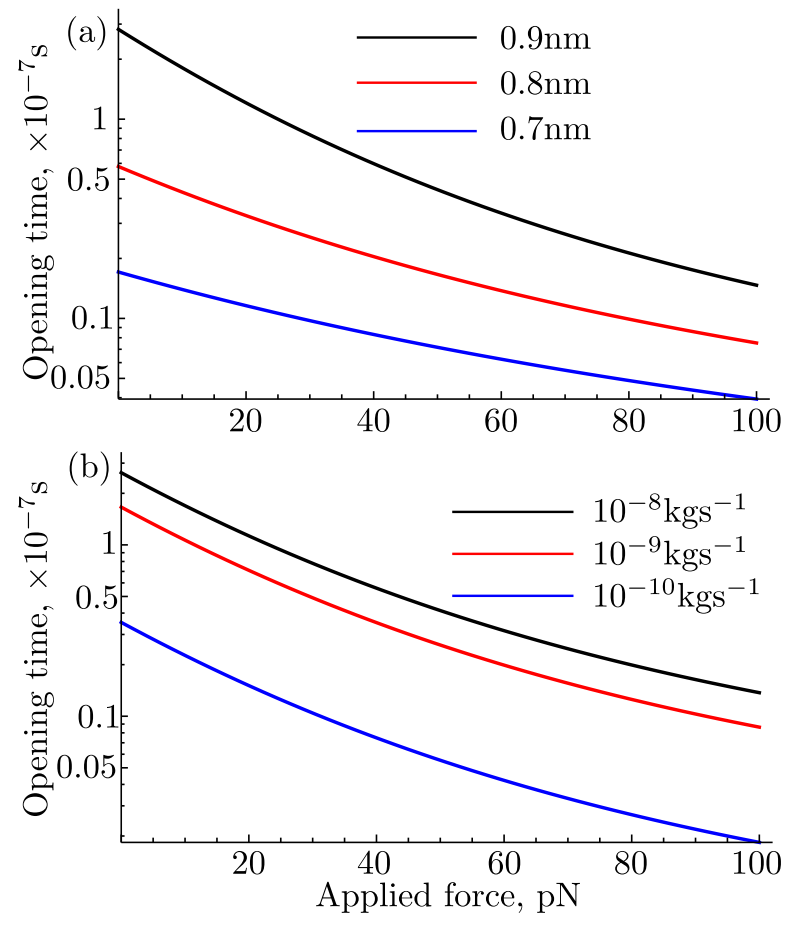

FIG. 4. MFPT for the two limiting cases plotted against applied force: (a) On a rigid substrate. The critical bond separation $u_{m}$ is different for the three curves: $u_{m}=0.7 \mathrm{~nm}$, $u_{m}=0.8 \mathrm{~nm}$, and $u_{m}=0.9 \mathrm{~nm}$, with the same energy barrier height $\Delta G_{0}$. This means that the bond is less stiff for longer breaking distance $u_{m}$; higher bond stiffness results in a smaller unbinding time. (b) For a viscous anchor, for different values of substrate friction $\gamma_{1}$, chosen to reflect the typical friction coefficients of polyacrylamide gels; a higher friction coefficient results in a longer opening time.

time, as the system takes longer to relax. Again, the unbinding time is monotonically decreasing as the force applied increases, although not in the exponential manner. Importantly, neither of these are simple exponential expressions with applied force, and so the simplest Bell model, $\tau \sim \exp \left[\left(\Delta G_{0}-f u_{m}\right) / k_{B} T\right]$, is not applicable.

Note that if either of the damping coefficients goes to zero, then the MFPT $\tau \rightarrow 0$ for both limits. The reason is the fluctuation-dissipation theorem: in the limit of vanishingly damping, the fluctuations in the system will be very large, and it will be knocked out over the barrier almost immediately. Of course, in such a limit, we are no longer free to neglect inertial terms. We will assume here, as in the extra-cellular matrix, that the fluid viscosity is much greater than the friction coefficient of the bond.

\section{FOKKER-PLANCK APPROACH}

If we want to consider more general viscoelastic substrates beyond the simple limits presented above, then there is no simple transformation of variables to find their separation. In particular, since the separation does not naturally emerge as a normal mode of the system, the formal solution has two exponential terms, and so cannot be expressed as a simple Ornstein-Uhlenbeck process. We have to find other ways to isolate the bond length, $u=x_{2}-x_{1}$, as the single variable. The approach explored here integrates out the second (fast-fluctuating) degree of freedom, and creates a 'mean field' approximation to the probability density, with the bond separation as the remaining single independent variable. To do this, we transform into a coordinate system based around the distance from the breaking point. Then we can integrate over lines of fixed distance from the barrier. Now we have a 1D problem, and can use the backward Fokker-Planck formalism to find the distribution of the first-passage times, and its mean.

Let us first write down the original diffusion (Smoluchowski) equation in the pair of $x_{1}, x_{2}$ coordinates, derived via standard methods from the underlying stochastic differential equations:

$$
\begin{aligned}
\frac{\partial p}{\partial t}= & D_{1} \frac{\partial^{2} p}{\partial x_{1}^{2}}+D_{2} \frac{\partial^{2} p}{\partial x_{2}^{2}} \\
& -\frac{\partial}{\partial x_{1}}\left[\left(\frac{\mu}{\gamma_{1}}\left(x_{2}-x_{1}\right)-\frac{\kappa x_{1}}{\gamma_{1}}\right) p\right] \\
& -\frac{\partial}{\partial x_{2}}\left[\left(-\frac{\mu}{\gamma_{2}}\left(x_{2}-x_{1}\right)+\frac{f}{\gamma_{2}}\right) p\right] .
\end{aligned}
$$

The coordinate transformation requires some algebra to manipulate, but it is natural to construct the new coordinates via: $u=x_{2}-x_{1}, \quad v=x_{1}+x_{2}$, where it is the $v$ variable that we are to integrate over to obtain a new diffusion equation for the effective probability distribution $P(u, t)=\int d v p(v, u)$. This relies on the behaviour of the full probability distribution and its derivative at $v= \pm \infty$, but since the potential is harmonic, we can safely assume that the probability distribution and its derivative go to zero faster than the potential becomes infinite. Then the mean-field diffusion equation in the single remaining variable $u$ takes the form:

$$
\frac{\partial P}{\partial t}=D \frac{\partial^{2} P}{\partial u^{2}}-\frac{\partial}{\partial u}\left[\left(-\frac{\tilde{\mu} u}{\tilde{\gamma}}+\frac{f}{\gamma_{2}}\right) P\right]
$$

where the effective stiffness of the bond $\tilde{\mu}=\mu+\kappa\left(\tilde{\gamma} / 2 \gamma_{1}\right)$ includes contributions from the bond and the substrate. Here the effective diffusion coefficient $D=D_{1}+D_{2}$ arises from using the Stokes-Einstein relation with the effective friction coefficient $\tilde{\gamma}$ :

$$
D=\frac{k_{B} T}{\gamma_{1}}+\frac{k_{B} T}{\gamma_{2}}=\frac{k_{B} T\left(\gamma_{1}+\gamma_{2}\right)}{\gamma_{1} \gamma_{2}}=\frac{k_{B} T}{\tilde{\gamma}}
$$

From Eq. 15 we can deduce the effective potential for the bond separation $u$ :

$$
V_{\mathrm{eff}}(u)=\frac{1}{2} \tilde{\mu} u^{2}-\frac{\tilde{\gamma}}{\gamma_{2}} f u,
$$


which contains a harmonic term combining the effects of the bond and of the substrate, and a linear term expressing the mechanical work done by the applied force $f$. This effective potential has the same shape as we saw in Fig. 2(b), but with significantly scaled parameters. For instance, the force is scaled by the factor $\tilde{\gamma} / \gamma_{2}$. There will be more discussion on this later, but we claim that this is the effective force felt across the bond at short times.

\section{Mean unfolding rate}

The effective potential in Eq. (17) could again allow us to treat this general case with the Ornstein-Uhlenbeck formalism, using the Ricciardi and Sato formalism. However, it is also possible to solve using the backward

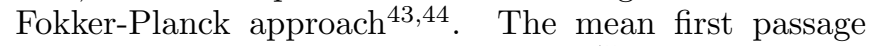
time for constant $D$ can be written as 45

$$
\tau\left(u_{0}\right)=\frac{1}{D} \int_{u_{0}}^{u_{m}} d y e^{\beta V_{\text {eff }}(y)} \int_{-\infty}^{y} d x e^{-\beta V_{\text {eff }}(x)},
$$

with $\beta=1 / k_{B} T$. This is for a specific initial condition $u(0)=u_{0}$, and so we must then average over the statistical distribution of starting positions for the overall characteristic time of unfolding:

$$
\tau=\int_{-\infty}^{u_{m}} p\left(u_{0}\right) \tau\left(u_{0}\right)
$$

The details of this calculation are found in the Appendix, but the result is the mean first-passage time for the breaking of the bond under a constant force $f$ :

$$
\begin{aligned}
\tau=\frac{\tilde{\gamma}}{\tilde{\mu}}\left(\frac{\sqrt{2 \pi / \beta \tilde{\mu}}}{u_{m}-\bar{u}}\right) & \exp \left[\frac{\beta \tilde{\mu}}{2}\left(u_{m}-\bar{u}\right)^{2}\right] \\
& \times\left(1-\exp \left[\frac{\beta \tilde{\mu}}{2}\left(\bar{u}^{2}-u_{m}^{2}\right)\right]\right),
\end{aligned}
$$

where $\bar{u}=f \tilde{\gamma} / \tilde{\mu} \gamma_{2}$ is the position of the effective free energy minimum shifted under the pulling force. The final bracket in this expression is important for when we surpass the critical force needed to destabilise the bond completely, it prevents the MFPT from diverging to $\infty$. However, it seems reasonable to only consider the regime well below the critical force,

$$
f<\frac{\tilde{\mu} u_{m} \gamma_{2}}{\tilde{\gamma}}=f_{c}
$$

Then we are free to drop the last bracketed term in 20 . At this stage the resulting expression becomes identical to the asymptotic form given by Ricciardi and Sato method in Eq. (6), which is a reassuring confirmation of its validity. The rate constant of unfolding is simply the
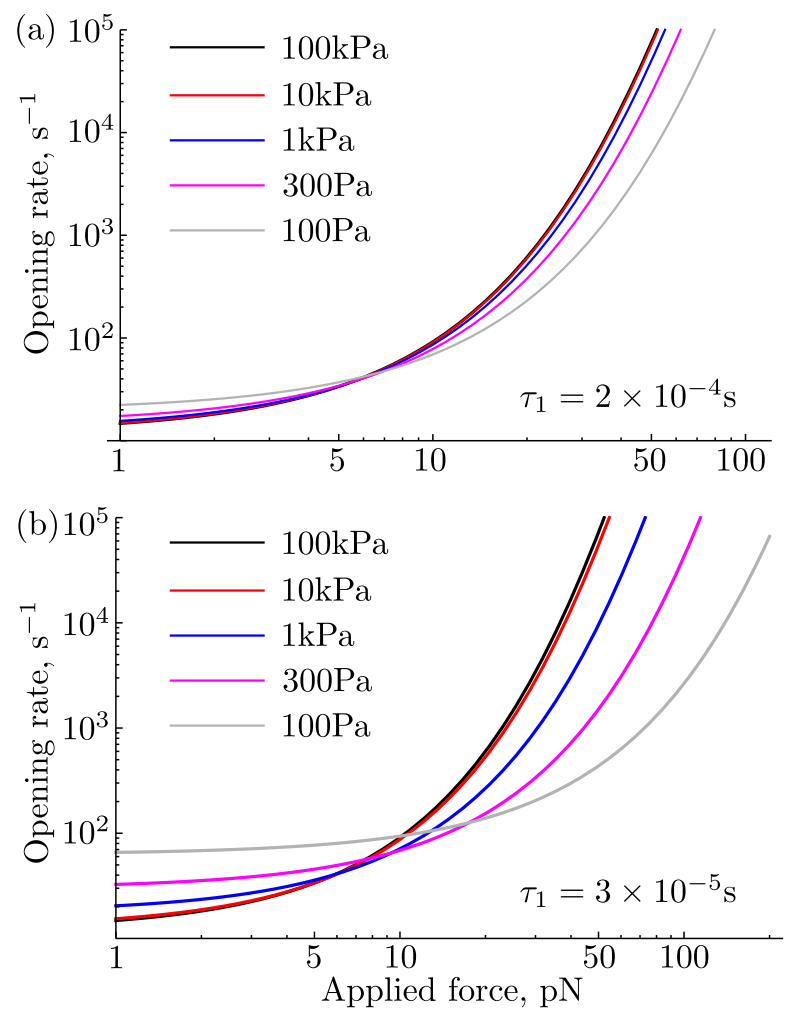

FIG. 5. The opening rate $k$ as a function of the applied force for substrates of different stiffness. (a) A longer relaxation time $\tau_{1}=2 \cdot 10^{-4} \mathrm{~s}$ reduces the sensitivity of the bond to substrate stiffness compared to the shorter relaxation time in (b). In both cases, the unfolding rate increases faster for stiffer substrates up to about $10 \mathrm{kPa}$, before the sensor response stops being stiffness-dependent. At low forces, the increased fluctuations in softer substrates increase the opening rate over stiffer substrates.

reciprocal of the MFPT:

$$
\begin{aligned}
& k \approx \frac{\tilde{\mu}}{\tilde{\gamma}}\left(\frac{u-f \tilde{\gamma} / \tilde{\mu} \gamma_{2}}{\sqrt{2 \pi k_{B} T / \tilde{\mu}}}\right) \\
& \quad \times \exp \left[-\beta\left(\Delta G_{0}+\frac{\kappa u_{m}^{2} \tilde{\gamma}^{2}}{8 \gamma_{1}^{2}}-f u \frac{\tilde{\gamma}}{\gamma_{2}}+\frac{f^{2}}{2 \tilde{\mu}}\left(\frac{\tilde{\gamma}}{\gamma_{2}}\right)^{2}\right)\right]
\end{aligned}
$$

where we substituted the expression for $\bar{u}$ to explicitly show the dependence on the pulling force $f$.

This expression for the rate of chain unfolding is quite intuitive. First of all, it scales with the overall relaxation rate of the effective mean field potential: $\tilde{\mu} / \tilde{\gamma}$. Then, it contains a factor comparing the distance from the free energy minimum to the barrier position $(u-\bar{u})$ with the characteristic length scale of thermal fluctuations within the energy barrier, $\sqrt{k_{B} T / \tilde{\mu}}$. Together, these two factors constitute the effective 'rate of attempts' of this stochastic process of bond-breaking. It is interesting that the rate prefactor is smaller when the equilibrium fluctuation around the free energy minimum is larger.

The remaining (activation) exponential factor is the most important effect at forces below the critical force (as 


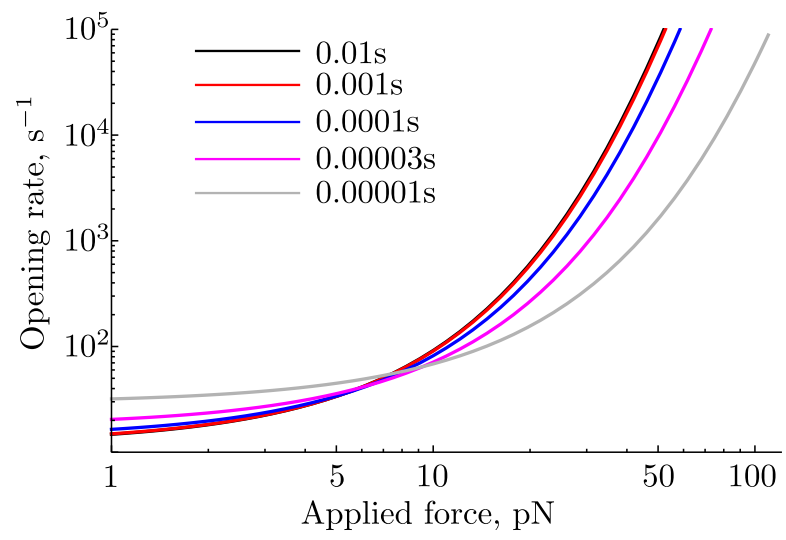

FIG. 6. The opening rate $k$ as a function of the applied force for a $1 \mathrm{kPa}$ substrate, for different substrate relaxation times $\tau_{1}$. Substrates with a shorter relaxation time have a lower rate of opening.

the second exponential in Eq. 201 will only contribute if $\left.\bar{u} \rightarrow u_{m}\right)$. Looking at the exponent in the rate expression 22, we can see a term coming from the zero-force energy barrier $\Delta G_{0}=\mu u_{m}^{2} / 2$, and an additional harmonic stretching term related to the substrate stiffness. At low forces, these factors will dominate the combined activation rate. The next term in the exponent is proportional to the reduction in the energy barrier due to the applied force, $-f u_{m}\left(\tilde{\gamma} / \gamma_{2}\right)$, which is essentially the modified 'Bell formula'. Finally, there is a term similar to the one seen in the earlier reaction path approach $23 \mid 46$ : the change in energy to the free energy minimum from the starting point: $-\left(f^{2} / 2 \tilde{\mu}\right)\left(\tilde{\gamma} / \gamma_{2}\right)^{2}$. This is an 'enzymatic effect' contributing to Arrhenius activation effect.

The variation of opening rate with applied force is plotted in Fig. 5. There is an increase in opening rate on stiffer substrates at higher forces, but the maximum is not within the physiological range of forces (around $100 \mathrm{pN}$ ). In reality, the rate constant should not decrease on further increase of the pulling force - this maximum only comes artificially as the steepest descent method loses its validity. In reality, the rate increase with the force remains monotonic. Secondly, there is a stiffness threshold, above which the complex is incapable of distinguishing substrates of different elastic modulus. For the parameters plotted here (a mesh size of $10 \mathrm{~nm}$ and a relaxation time of $2 \cdot 10^{-4} \mathrm{~s}$ ), this stiffness threshold is around $10 \mathrm{kPa}$, but depends strongly on the substrate's viscoelastic properties. This is the same stiffness that Yeung et al. found a plateau in cell circumference with increasing stiffness ${ }^{39}$, and it also is very close to the stiffness threshold for myofibroblast differentiation $\frac{47}{}$.

Figure 6 shows how the relaxation time of the substrate influences the rate of chain unfolding. Longer relaxation times lead to higher opening rates. The rate of opening given in Eq. (22) shows some interesting features. First of all, the stiffness $\kappa$ always comes with a scaling factor $\tilde{\gamma} / \gamma_{1}=\gamma_{2} /\left(\gamma_{1}+\gamma_{2}\right)$. As such, if $\gamma_{1} \gg \gamma_{2}$, then the scaling factor is zero, and there is no direct dependence of the opening rate on the substrate stiffness (of course, in our study, there is an indirect relationship through the substrate relaxation time $\tau_{1}$ ). Indeed, for a substrate with a long relaxation time, $\tau_{1}=0.01 \mathrm{~s}$, there is no sensitivity to substrate stiffness at all. This sensitivity is controlled by the balance between the viscous properties of the substrate and those of the bond. When $\gamma_{1} \sim \gamma_{2}$, increasing the substrate stiffness increases the effective stiffness of the bond in the bond length variable. This has the effect of diminishing the contribution from the $f^{2}$ term in the exponent, and increasing the bare rate $\tilde{\mu} / \tilde{\gamma}$.

If we take a rough force value of $\sim 30 \mathrm{pN}$ across each integrin-ECM bond $\frac{48}{4}$, then by Fig. 6, we are in the region where greater substrate stress relaxation leads to a higher response from the mechanosensing molecule. This is in accord with experimental results of cells spreading on viscoelastic substrates, where spreading is enhanced on substrates with greater stress relaxation $\frac{49}{}$.

\section{DISCUSSION}

First of all, we need to emphasize (or remind) that by the 'rate of unfolding' (or 'opening rate') here we mean the inverse of the average time when the folded chain undergoes the abrupt and complete unfolding under a constant pulling force, since that is the process in the Gibbs ensemble ${ }^{9}$ 11. The current approach to the calculation of this MFPT, using the mean-field reduction to the singlevariable stochastic process and the implicit summation over the reaction pathways in the $\left(x_{1}, x_{2}\right)$ space, is shown to be reliable and produces the physically sensible result. First, the unfolding rate in the mean field approach is monotonic, compared to a low maximum for the earlier reaction-path approach. The reason for this is clear: when we take a specific two-part reaction path, the overall rate is controlled by the time to reach the minimum along the substrate coordinate $x_{1}$, and it is assumed that the system does not reach the minimum in $u$ space before it reaches the minimum in $x_{1}$ space.

To investigate this, we can plot the deterministic force expected across the bond, without any stochastic effects, using the equations of motion:

$$
\begin{aligned}
& \gamma_{1} \dot{x_{1}}(t)=-\kappa x_{1}(t)-\frac{\partial U\left(x_{2}(t)-x_{1}(t)\right)}{\partial x_{1}} \\
& \gamma_{2} \dot{x_{2}}(t)=-\frac{\partial U\left(x_{2}(t)-x_{1}(t)\right)}{\partial x_{2}}+f
\end{aligned}
$$

The full solution of this set of ordinary differential equations for $x_{1}(t)$ and $x_{2}(t)$, with a harmonic potential $U=\mu u^{2} / 2$, is straightforward. We could then examine the bond separation, $u=x_{2}(t)-x_{1}(t)$ as a function of time; the full expression is unwieldy, but an intuitive approximation is

$$
u(t)=\frac{f}{\mu}\left(\frac{\tilde{\gamma}}{\gamma_{2}}\left[1-e^{-\tilde{\mu} t / \tilde{\gamma}}\right]+\frac{\tilde{\gamma}}{\gamma_{1}}\left[1-e^{-t / \tau_{1}}\right]\right),
$$




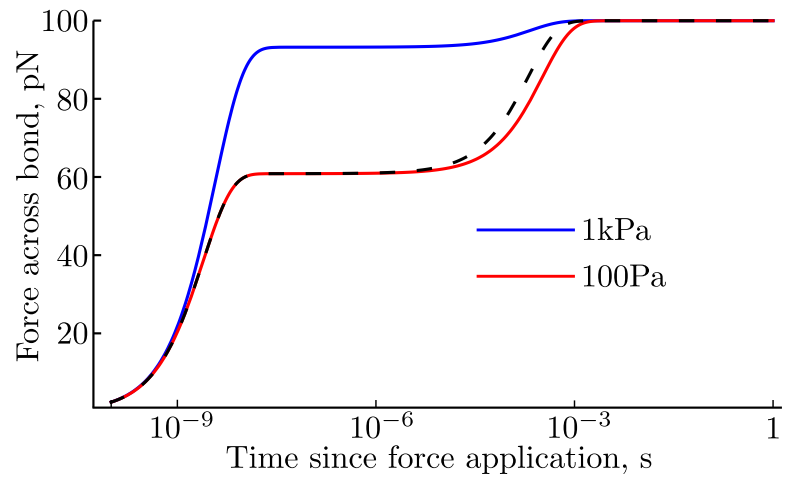

FIG. 7. The evolution of deterministic force $\mu u(t)$ across the bond upon application of an external pulling force $f=100 \mathrm{pN}$ at $t=0$, plotted for two different substrate moduli. There is an initial rise in the force to an intermediate threshold, and then a slower increase up to the full value of $f$ (here, $\tau_{\text {sub }}=2 \cdot 10^{-4} \mathrm{~s}$ ). This threshold is a balance of the friction coefficients in the bond and in the substrate, and is lower for the softer substrate (this has a lower coefficient of friction). The approximation in Eq. (24) is plotted for $100 \mathrm{~Pa}$ as the dashed black curve; the same approximate expression lies directly on top of the $1 \mathrm{kPa}$ curve.

and plot the actual force acting across the bond: $\mu u(t)$, see Fig. 7. For long substrate relaxation times, the extension rapidly reaches an intermediate free energy minimum $f \tilde{\gamma} / \mu \gamma_{2}$, extending further as the substrates slowly reaches its full extension. Importantly, if the force is larger than the critical force so that the intermediate minimum is past the barrier, $f \tilde{\gamma} / \mu \gamma_{2}>u$, then the bond will break in this initial extension phase. There is no reduction in the unfolding rate as the external pulling force goes to infinity; the deterministic breaking time above the critical force is a monotonically increasing function with force. This interpretation explains the presence of the factor $\tilde{\gamma} / \gamma_{2}$ in the mean-field Arrhenius factor: on the timescale of the MFPT, the relevant value of force across the bond is this scaled one. For typical polyacrylamide gels, this factor is only relevant for soft substrates $(<1 \mathrm{kPa})$. The plot of deterministic extension shows that the reaction-path approach ${ }^{23}$ does not capture essential parts of the physics at high forces. During the first leg of the movement, there appears to be an assumption that there is no effective force across the bond facilitating the opening event, and Fig. 7 shows this notion to be false; we build up some value of force across the bond very quickly, and it is this that 'tests' the bond for opening.

It may well be that the 'mean field' approach taken here, based on the integrating out the redundant variable, provides a route to solving the problem for more general viscoelastic substrates. In particular, if the internal relaxation in the substrate is slow compared to the typical breaking time of the bond, then it may be possible to treat the substrate as quasi-stationary, with an effective constant force across the bond. Remember that as long as we can find the overall effective friction co- efficient on the reaction timescale, and the effective force, then we can use the Ricciardi-Sato formula to obtain the mean first passage time quickly.

How could the reaction rates calculated here for the unfolding of molecules, such as FAK or talin, be included in a cellular description of mechanosensing? One prominent description of focal adhesion dynamics is given by the 'clutch model', which couples an internal mechanosensing molecule, with the on/off rates of integrin binding to the ECM (for a recent survey, see for example ${ }^{\sqrt[50]{0}}$ ). Integrin is a catch-bond: as force is applied to integrins, the strength of its bond with ECM initially increases, in contrast to the usual trend of biomolecules to become less stable with increasing force 5152 . Above a certain force, the bond strength starts to decrease, in line with 'conventional' bond dynamics described by the Bell mode 20 . The interplay between the two unbinding/unfolding timescales allow the cell to distinguish between stiff and soft substrates.

However, such molecules as FAK and talin not only act as force transmitters (to pass the cytoskeletal pull down to integrin-ECM bond), but also force transducers, initiating signalling pathways throughout the cell. To successfully model these, it is likely that a complex kinetic model is needed, accounting for activation of signalling pathways, turnover of focal adhesion units (and therefore active force transducers), and the feedback mechanisms on e.g. cytoskeletal force changes as a result of downstream signalling events. Such a kinetic model is very sensitive to the rates of its component processes, and the work in this paper is well placed to inform the activation rates of force transducers in such models. In this context, the rate constants calculated here provide more complete expressions for the unfolding rates of the internal mechanosensor, and the dependence upon substrate viscoelasticity, which has not yet been fully addressed in the literature.

\section{CONCLUSIONS}

To conclude, we have shown that the unfolding rates of proteins depend on the viscoelastic properties of the substrate they are tethered to. In particular, when the dissipation in the substrate is closely tuned to the dissipation in the bond, there is a regime where substantially reduced force may be felt across the unfolding bond as the substrate relaxes. In this case, lower stiffness substrates (longer relaxation times) lead to a reduced unfolding rate above a force threshold ( $\sim 10 \mathrm{pN}$ in our model). Below this force threshold, the enhanced fluctuations in soft substrates increases the unfolding rate over stiff substrates. We are not aware of any studies which would allow quantitative comparison with experiments, but the results of this study have some qualitative commonalities with biological experiments of stiffness sensing. On a basic level, there is a direct correspondence between the increase in unfolding rate on a stiffer substrate (at 
medium to high forces), and the observation that cells spread further, with more developed stress fibres and more stable focal adhesions, on stiffer substrates than soft ones 39153154 .

Another point is that substrates with a modulus above $1-10 \mathrm{kPa}$ appear as 'stiff' for spreading cells (compare with Fig. 5], $\frac{39}{3}$ with broad variations between cell types, e.g. endothelial cells and fibroblasts, all using the same integrin complex, possibly explained by a variation in $\tau_{1}$ in their natural environment. In many in-vitro experiments on protein unfolding by an AFM or tweezerapplied force (see, for example $e^{9|10| 55 \mid 56}$ ), the reported results invariably confirm the exponential 'Bell-like' increase of the rate with force, but the authors never attempted fitting the expression like our main Eq. (22), where the additional quadratic force appears in the exponent when the substrate stiffness $\kappa$ is low, or the constant barrier expression is renormalised. It would be promising to re-analyse those data sets from this point of view.

Further, the interpretation offered here, to consider the effective force felt across the bond as quasi-static during substrate relaxation, may allow more complicated viscoelastic models to be analysed in the future, allowing for more realistic modelling both of mechanosensing processes in the molecular clutch model, and for AFM/optical tweezers pulling experiments.

\section{ACKNOWLEDGEMENTS}

The authors have benefited from useful conversations with Cheng-Tai Lee and Jonathan Eddyshaw, as well as useful discussion with Sergi Garcia-Manyes on the application to experiments. This work has been funded by EPSRC EP/M508007/1.

\section{REFERENCES}

${ }^{1}$ S. Kumar and M. S. Li, "Biomolecules under mechanical force," Phys. Rep. 486, 1-74 (2010).

${ }^{2}$ P. Baláž, M. Achimovičová, M. Baláž, P. Billik, Z. CherkezovaZheleva, and et al., "Hallmarks of mechanochemistry: from nanoparticles to technology," Chem. Soc. Rev. 42, 7571-7637 (2013).

${ }^{3}$ J. L. Howard, Q. Cao, and D. L. Browne, "Mechanochemistry as an emerging tool for molecular synthesis: what can it offer?" Chem. Sci. 9, 3080-3094 (2018).

${ }^{4}$ A. Engel, H. Gaub, and D. Müller, "Atomic force microscopy: A forceful way with single molecules," Curr. Biol. 9, R133-R136 (1999)

${ }^{5}$ T. E. Fisher, A. F. Oberhauser, M. Carrion-Vazquez, P. E. Marszalek, and J. M. Fernandez, "The study of protein mechanics with the atomic force microscope," Trends Biochem. Sci. 24, 379-384 (1999).

${ }^{6}$ C. Bustamante, Z. Bryant, and S. B. Smith, "Ten years of tension: single-molecule dna mechanics." Nature 421, 423-7 (2003).

${ }^{7}$ M. Rief, "Reversible unfolding of individual titin immunoglobulin domains by AFM," Science 276, 1109-1112 (1997).

${ }^{8}$ S. B. Fowler, R. B. Best, J. L. Toca Herrera, T. J. Rutherford, A. Steward, E. Paci, M. Karplus, and J. Clarke, "Mechanical unfolding of a titin ig domain: structure of unfolding intermediate revealed by combining afm, molecular dynamics simulations, nmr and protein engineering." J. Mol. Biol. 322, 841-849 (2002).

${ }^{9}$ A. F. Oberhauser, P. K. Hansma, M. Carrion-Vazquez, and J. M. Fernandez, "Stepwise unfolding of titin under force-clamp atomic force microscopy," Proc. Natl. Acad. Sci. USA 98, 468$472(2001)$

${ }^{10}$ S. Garcia-Manyes, J. Brujić, C. L. Badilla, and J. M. Fernández, "Force-clamp spectroscopy of single-protein monomers reveals the individual unfolding and folding pathways of i27 and ubiquitin," Biophys. J. 93, 2436-46 (2007).

${ }^{11} \mathrm{~S}$. Bell and E. M. Terentjev, "Unfolding of globular polymers by external force," J. Chem. Phys. 143, 184902 (2015)

12 A. D. Bershadsky, M. Kozlov, and B. Geiger, "Adhesionmediated mechanosensitivity: a time to experiment, and a time to theorize," Curr. Opin. Cell Biol. 18, 472-481 (2006).

${ }^{13}$ B. Geiger, J. P. Spatz, and A. D. Bershadsky, "Environmental sensing through focal adhesions," Nat. Rev. Mol. Cell Biol. 10, 21-33 (2009).

${ }^{14}$ M. Cockerill, M. K. Rigozzi, and E. M. Terentjev, "Mechanosensitivity of the 2nd kind: Tgf- $\beta$ mechanism of cell sensing the substrate stiffness," PloS One 10, e0139959 (2015).

${ }^{15}$ L. R. Opsahl and W. W. Webb, "Transduction of membrane tension by the ion channel alamethicin," Biophys. J. 66, 71-74 (1994).

${ }^{16}$ F. Sachs, "Stretch-activated ion channels: what are they?" Physiology 25, 50-56 (2010).

${ }^{17}$ E. S. Haswell, R. Phillips, and D. C. Rees, "Mechanosensitive channels: what can they do and how do they do it?" Structure 19, 1356-1369 (2011).

${ }^{18}$ E. Evans and K. Ritchie, "Dynamic strength of molecular adhesion bonds," Biophys. J. 72, 1541-1555 (1997).

${ }^{19} \mathrm{R}$. Bruinsma, "Theory of force regulation by nascent adhesion sites," Biophys. J. 89, 87-94 (2005).

${ }^{20}$ G. I. Bell, "Models for the specific adhesion of cells to cells," Science 200, 618-627 (1978).

${ }^{21} \mathrm{H}$. A. Kramers, "Brownian motion in a field of force and the diffusion model of chemical reactions," Physica 7, 284-304 (1940).

${ }^{22}$ P. Hanggi, "Brownian motion in the field of force," J. Stat. Phys. 42, 105-148 (1986).

${ }^{23} \mathrm{~S}$. Bell and E. M. Terentjev, "Focal adhesion kinase: the reversible molecular mechanosensor," Biophys. J. 112, 2439-2450 (2017).

${ }^{24}$ M. D. Schaller, C. A. Borgman, B. S. Cobb, R. R. Vines, A. B. Reynolds, and J. T. Parsons, "pp125-fak, a structurally distinctive protein-tyrosine kinase associated with focal adhesions," Proc. Natl. Acad. Sci. USA 89, 5192-5196 (1992).

${ }^{25}$ J. Marko and E. Siggia, "Stretching dna," Macromolecules 28, 8759-8770 (1995).

${ }^{26}$ J. Zhou, C. Aponte-Santamaría, S. Sturm, J. T. Bullerjahn, A. Bronowska, and F. Gräter, "Mechanism of focal adhesion kinase mechanosensing," PLoS Comp. Biol. 11, e1004593 (2015).

${ }^{27}$ R. M. Bhaskara and N. Srinivasan, "Stability of domain structures in multi-domain proteins," Sci. Rep. 1, 40 (2011).

${ }^{28}$ M. D. Brenner, R. Zhou, and H. T., "Forcing a connection: impacts of single-molecule force spectroscopy on in vivo tension sensing," Biopolymers 95, 332-344 (2011).

${ }^{29}$ T. Lecuit, P. F. Lenne, and E. Munro, "Force generation, transmission, and integration during cell and tissue morphogenesis," Annu. Rev. Cell Dev. Biol. 27, 157-184 (2011).

${ }^{30}$ J. T. Finer, R. M. Simmons, and J. A. Spudich, "Single myosin molecular mechanics: piconewton forces and nanometer steps," Nature 368, 113-119 (1994).

${ }^{31}$ A. D. Bershadsky, N. Q. Balaban, and B. Geiger, "Adhesiondependent cell mechanosensitivity," Annu. Rev. Cell Dev. Biol. 19, 677-695 (2003).

${ }^{32}$ C. M. Pollock and R. E. Shadwick, "Relationship between body mass and biomechanical properties of limb tendons in adult mammals," Am. J. Physiol. 266, R1016-R1021 (1994). 
${ }^{33}$ J. M. Gosline, M. A. Lillie, E. Carrington, P. Guerette, C. Ortlepp, and K. Savage, "Elastic proteins: biological roles and mechanical properties," Philos. Trans. R. Soc. Lond. B Biol. Sci. 357, 121-132 (2002).

${ }^{34}$ C. M. Bellingham, M. A. Lillie, J. M. Gosline, G. M. Wright, B. C. Starcher, A. J. Bailey, K. A. Woodhouse, and F. W. Keeley, "Recombinant human elastin polypeptides self-assemble into biomaterials with elastin-like properties," Biopolymers 70, 445455 (2003).

${ }^{35}$ S. M. Mithieux, J. E. J. Rasko, and A. S. Weiss, "Synthetic elastin hydrogels derived from massive elastic assemblies of selforganized human protein monomers," Biomaterials 25, 49214927 (2004)

${ }^{36}$ K. S. Kolahi, A. Donjacour, X. Liu, W. Lin, R. K. Simbulan, E. Bloise, E. Maltepe, and P. Rinaudo, "Effect of substrate stiffness on early mouse embryo development," PloS One 7, e41717 (2012).

${ }^{37}$ A. M. Handorf, Y. Zhou, M. A. Halanski, and W.-J. Li, "Tissue stiffness dictates development, homeostasis, and disease progression," Organogenesis 11, 1-15 (2015).

${ }^{38}$ L. D. Landau and I. M. Lifshitz, Theory of Elasticity, 3rd ed. (Butterworth-Heinemann, Oxford, 1986).

${ }^{39}$ T. Yeung, P. Georges, L. A. Flanagan, B. Marg, M. Ortiz, M. Funaki, N. Zahir, W. Ming, V. Weaver, and P. Janmey, "Effects of subtrate stiffness on cell morphology, cytoskeletal structure, and adhesion," Cell Motil. Cytoskeleton 60, 24-34 (2005).

${ }^{40}$ U. Mayor, C. M. Johnson, V. Daggett, and A. R. Fersht, "Protein folding and unfolding in microseconds to nanoseconds by experiment and simulation," Proc. Natl. Acad. Sci. USA 97, 13518-13522 (2000).

${ }^{41}$ Y. Abidine, V. M. Laurent, R. Michel, A. Duperray, L. I. Palade, and C. Verdier, "Physical properties of polyacrylamide gels probed by afm and rheology," EPL 109, 38003 (2015).

${ }^{42}$ L. M. Ricciardi and S. Sato, "First-passage-time density and moments of the ornstein-uhlenbeck process," J. Appl. Prob. 25, 4357 (1988).

${ }^{43}$ R. Zwanzig, Nonequilibrium Statistical Mechanics (Oxford University Press, New York, 2001).

${ }^{44}$ R. W. Pastor, R. Zwanzig, and A. Szabo, "Diffusion limited first contact of the ends of a polymer: comparison of theory with simulation," J. Chem. Phys. 105, 3878-3882 (1996).

${ }^{45}$ A. Szabo, K. Schulten, and Z. Schulten, "First passage time approach to diffusion controlled reactions," J. Chem. Phys. 72, 4350-4357 (1980).

${ }^{46}$ M. Escudé, M. K. Rigozzi, and E. M. Terentjev, "How cells feel: Stochastic model for a molecular mechanosensor," Biophys. J. 106, 124-133 (2014).

${ }^{47}$ P. Wipff, D. Rifkin, J.-J. Meister, and B. Hinz, "Myofibroblast contraction activates latent tgf- $\beta 1$ from the extracellular matrix," J. Cell Biol. 179, 1311-1323 (2007).

${ }^{48}$ S. W. Moore, P. Roca-Cusachs, and M. P. Sheetz, "Stretchy proteins on stretchy substrates: the important elements of integrinmediated rigidity sensing," Dev. Cell 19, 194-206 (2010).

${ }^{49}$ O. Chaudhuri, L. Gu, M. Darnell, D. Klumpers, S. A. Bencherif, J. C. Weaver, N. Huebsch, and D. J. Mooney, "Substrate stress relaxation regulates cell spreading," Nat. Comms. 6, 6364 (2015).

${ }^{50}$ A. Elosegui-Artola, X. Trepat, and P. Roca-Cusachs, "Control of mechanotransduction by molecular clutch dynamics," Trends Cell. Biol. 28, 356-367 (2018).

${ }^{51}$ E. A. Novikova and C. Storm, "Contractile fibers and catch-bond clusters: a biological force sensor?" Biophys. J. 105, 1336-1345 (2013).

${ }^{52}$ F. Kong, A. J. Garcia, A. P. Mould, M. J. Humphries, and C. Zhu, "Demonstration of catch bonds between an integrin and its ligand," J. Cell Biol. 185, 1275-1284 (2009).

${ }^{53}$ D. E. Discher, P. Janmey, and Y.-L. Wang, "Tissue cells feel and respond to the stiffness of their substrate," Science 310, 11391143 (2005).

${ }^{54}$ R. J. Pelham and Y.-L. Wang, "Cell locomotion and focal adhesions are regulated by substrate flexibility," Proc. Natl Acad.
Sci. USA 94, 13661-13665 (1997).

${ }^{55}$ M. Carrion-Vazquez, A. F. Oberhauser, S. B. Fowler, P. E. Marszalek, S. E. Broedel, J. Clarke, and J. M. Fernandez, "Mechanical and chemical unfolding of a single protein: A comparison," Proc. Natl. Acad. Sci. USA 96, 3694-3699 (1999).

${ }^{56} \mathrm{M}$. Schlierf, H. Li, and J. M. Fernandez, "The unfolding kinetics of ubiquitin captured with single-molecule force-clamp techniques," Proc. Natl Acad. Sci. USA 101, 7299-7304 (2004).

\section{Appendix A: Backward F-P approach to MFPT}

The inner integral in Eq. 18 can be rewritten as the cumulative density of the normal distribution, essentially given by the error function $\Phi(x)$, scaled by a factor:

$$
\tau\left(u_{0}\right)=\tilde{\gamma} \sqrt{\frac{2 \pi \beta}{\tilde{\mu}}} \int_{u_{0}}^{u_{m}} d y e^{\beta(y-\bar{u})^{2} / 2} \Phi(\sqrt{\beta \tilde{\mu}}(y-\bar{u}))
$$

This integral is not analytically solvable, however, the steepest descent makes a good approximation. In particular, we can write the integrand as an exponential

$$
\tau\left(u_{0}\right)=\tilde{\gamma} \sqrt{\frac{2 \pi \beta}{\tilde{\mu}}} \int_{-u_{m}}^{u_{0}} d y e^{\Psi(y)}
$$

where the function $\Psi(y)$ is

$$
\Psi(y)=\frac{\beta \tilde{\mu}(y-\bar{u})^{2}}{2}+\ln \Phi(\sqrt{\beta \tilde{\mu}}(y-\bar{u})) .
$$

The exponent $\Psi(y)$ is plotted in Fig. 8, and it is evidently dominated by the region around the barrier, at $u \rightarrow u_{m}$. As such, we can expand the exponent around the barrier: $\Psi(y) \approx \Psi\left(u_{m}\right)+\Psi^{\prime}\left(u_{m}\right)\left(y-u_{m}\right)$, and the mean passage time from $u_{0}$ to $u_{m}$ can be approximated as

$$
\begin{aligned}
\tau\left(u_{0}\right) & =\tilde{\gamma} \sqrt{\frac{2 \pi \beta}{\tilde{\mu}}} e^{\Psi\left(u_{m}\right)-\Psi^{\prime}\left(u_{m}\right) u_{m}} \int_{u_{0}}^{u_{m}} e^{\Psi^{\prime}\left(u_{m}\right) y} d y \\
& =\tilde{\gamma} \sqrt{\frac{2 \pi \beta}{\tilde{\mu}}} \frac{e^{\Psi\left(u_{m}\right)}}{\Psi^{\prime}\left(u_{m}\right)}\left(1-e^{\Psi^{\prime}\left(u_{m}\right)\left(u_{0}-u_{m}\right)}\right) .
\end{aligned}
$$

We are now close to an answer, and all that is left is to average over the initial position distribution, which we take to be the force-free steady state:

$$
p\left(u_{0}\right)=\sqrt{\frac{\beta \tilde{\mu}}{2 \pi}} \exp \left[-\beta \tilde{\mu} \frac{u_{0}^{2}}{2}\right] .
$$

The probability $p\left(u_{0}\right)$ decays sufficiently fast away from $u_{0}=0$ that we can extend the lower limit of integration to infinity, past the barrier limit. In the averaging of $\tau\left(u_{0}\right)$ we only have to deal with the integral

$$
\sqrt{\frac{\beta \tilde{\mu}}{2 \pi}} \int_{-\infty}^{\infty} e^{-\beta \tilde{\mu} u_{0}^{2} / 2+\Psi^{\prime}\left(u_{m}\right) u_{0}} d u_{0}=e^{\Psi^{\prime}\left(u_{m}\right)^{2} / 2 \beta \tilde{\mu}} .
$$




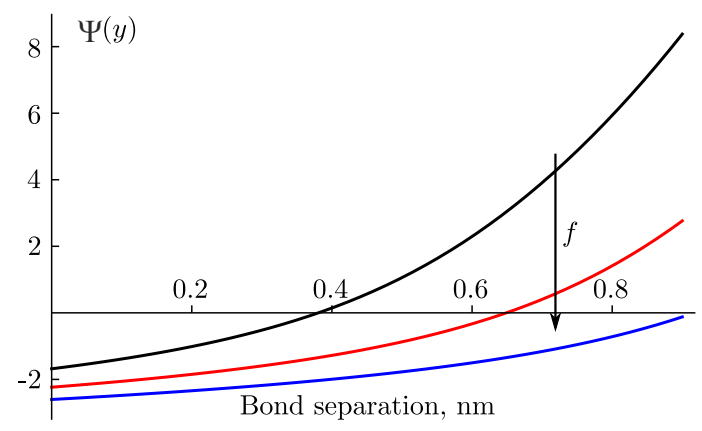

FIG. 8. The exponent of the steepest descent $\Psi(y)$ plotted as a function of the bond separation $u$, for a maximal bond length of $u_{m}=0.9 \mathrm{~nm}$ on a $1 \mathrm{kPa}$ substrate. The three curves are for the increasing applied force $f=50 \mathrm{pN}$ (black curve), $f=100 \mathrm{pN}$ (red curve) and $f=150 \mathrm{pN}$ (blue curve). For low forces, the exponent is very large close to the barrier, and so the integral in Eq. A2 will be well approximated by the method of steepest descent around the barrier. However, as the force nears the critical force, at around $150 \mathrm{pN}$, this approximation is much less sound.
Finally, the full mean first passage time is:

$$
\tau=\tilde{\gamma} \sqrt{\frac{2 \pi \beta}{\tilde{\mu}}} \frac{e^{\Psi\left(u_{m}\right)}}{\Psi^{\prime}\left(u_{m}\right)}\left(1-e^{\Psi^{\prime}\left(u_{m}\right)^{2} / 2 \beta \tilde{\mu}-\Psi^{\prime}\left(u_{m}\right) u_{m}}\right)
$$

Usefully, the function $\Phi(\sqrt{\beta \tilde{\mu}}(y-\bar{u})$ can be neglected: when the force is below the critical force $f / \tilde{\mu}<u \gamma_{2} / \tilde{\gamma}$, $\Phi(\sqrt{\beta \tilde{\mu}}(y-\bar{u}) \approx 1$. In this limit, we can write down the mean first passage time in a compact analytical form, which is given in the Eq. 20) in main text. 